calculation (laboratory full blood count; LHCT) and conductivity (blood gas analyzer haematocrit; BGHCT). All three methods are often used interchangeably or simultaneously. However, limited studies suggest that SHCT values are higher than others due to plasma trapping. This study compares neonatal HCT measurements by the three different techniques using statistical methods.

Methods Retrospective review of data extracted from a point of care testing logbook kept in our neonatal unit (for SHCT and BGHCT) and electronic laboratory records. Venous samples that concurrent testing with more than one methodology was done were included. Capillary and arterial samples were excluded. Results from each method were compared using a paired sample t-test and agreement was examined by BlandAltman analysis.

Results A total of 137 samples from 127 unique neonatal patients were identified. The mean and median HCT values for each method are shown in table 1. A strong correlation $\left(\mathrm{R}^{2}=0.875\right)$ between LHCT and SHCT values, as well as between LHCT and BGHCT values $\left(\mathrm{R}^{2}=0.903\right)$ was seen. However, SHCT values were higher than LHCT values with a mean difference of $5.19 \%(4.53,5.86)$. A paired t-test found this difference to be statistically significant; $\mathrm{t}(126)=15.5$, $\mathrm{p}<0.001$. This mean difference was even higher $(6.7 \%)$ in the subgroup analysis for HCT values $>65 \%$ which are consistent with polycythemia. The Bland-Altman difference plot for LHCT and SHCT methods demonstrated a good agreement between the two, but also defined a bias of $5.2 \%$, with an agreement range of -2.18 to 12.57 .

\begin{tabular}{llll}
\multicolumn{2}{l}{ Abstract G571 Table 1} & & \\
\hline & Median & Mean & SD \\
\hline SHCT & 59 & 58.98 & 10.45 \\
LHCT & 54 & 53.35 & 9.25 \\
BGHCT & 57 & 55 & 11.7 \\
\hline
\end{tabular}

Conclusion Although there is good correlation between all the test values, our study confirms that SHCT values are consistently higher than the others. Further studies are needed to investigate the clinical impact of this observed difference between methodologies, in neonatal pratice and particularly in the management of neonatal polycythemia.

\section{G572(P) EVIDENCE FOR FEEDING PRACTICES IN VERY LOW BIRTHWEIGHT INFANTS IN SUB-SAHARAN AFRICA AND IMPLICATIONS FOR FUTURE RESEARCH: AN OVERVIEW OF SYSTEMATIC REVIEWS}

${ }^{1} \mathrm{AE}$ Akindolire, ${ }^{2} \mathrm{~A}$ Talbert, ${ }^{3} \mathrm{~S} J$ Allen, ${ }^{3}$ The Neonatal Nutrition Network. ${ }^{1}$ Paediatrics, University of Ibadan/University College Hospital, Ibadan, Nigeria; ${ }^{2}$ KEMRIWellcome, Kilifi, Kenya; ${ }^{3}$ Neonatal Nutrition Network, Liverpool School of Tropical Medicine, Liverpool, UK

\subsection{6/archdischild-2020-rcpch.490}

Aim To review the evidence available for optimising feeding of VLBW infants in sub Saharan Africa (sSA) and to identify areas for future research.

Methods We did a comprehensive literature search in Cochrane Database of Systematic reviews, EMBASE, PUBMED and CINAHL (July 2019). The key search terms were: very low birthweight AND (enteral feeding or enteral nutrition) AND (systematic review or meta-analysis). Systematic reviews and meta-analyses of randomised and quasi-randomised controlled trials of interventions which focused on the effect of four 'basic' feeding practices: when to start enteral feeds, what to feed when EBM is insufficient or unavailable, how to feed and how to advance feeds on weight gain during hospital stay and time to achieve full enteral feeds were eligible for inclusion. We assessed the quality of each review using the AMSTAR (A Measurement Tool to Assess systematic Reviews) tool.

Results We included 8 systematic reviews comprising of 10 comparisons, all were of high methodological quality and assessed what to feed, how to feed and how to advance feeds. There were no reviews which examined the effect of early trophic feeding (at age $<24$ hours) and the use of preterm formula. There was no clear evidence of benefit of fortification of breast milk from the 3 reviews assessed. There was no clear evidence of benefit of use of nasogastric versus orogastric feeding and continuous versus bolus feeds. Rapid advancement of feeds was found to reduce time to full enteral feeds and episodes of late onset sepsis. None of the trials were done in sSA.

Conclusion There is a need for pragmatic trials in preterm feeding in sub-Saharan Africa that would consider inexpensive, low technology and sustainable interventions in the area of when to start feeds, what to feed, absolute volumes, modes of feeding and use of fortification. There is evidence that rapid advancement of feeds will confer benefits to babies in sSA where fortification with extra protein or calories might not be feasible, affordable or available.

\section{G573(P) 'HOW LONG IS NOT SHORT?': DURATION OF CAFFEINE THERAPY IN PRETERM INFANTS - A RANDOMIZED CONTROLLED TRIAL}

R Prakash, F Pournami, J Prabhakar, A Nandakumar, PMC Nair, N Jain. Department of Neonatology, Kerala Institute of Medical Sciences, Trivandrum, India

\subsection{6/archdischild-2020-rcpch.491}

Aim The aim was to compare recurrence of clinically significant apnea (CSA) using 2 protocols to stop caffeine therapy in preterm infants.

Methods This prospective, randomized controlled trial studied babies born at 26-32 weeks gestation;in 2 groups: Group 1 (SEVEN): Caffeine therapy was stopped immediately once the infants on caffeine were apnea free and off respiratory supports for 7 consecutive days; Group 2 (THIRTYFOUR): Caffeine therapy was continued mandatorily till minimum of 34 weeks postmentrual age(PMA) and thereafter stopped only after baby was apnea free and off respiratory supports for at least one week. Randomization was done using a computer generated random number sequence and allocation concealment using Sequentially Numbered Opaque Sealed Envelopes. The predefined primary outcome was proportion of infants with recurrence of clinically significant apnea after stopping caffeine. The secondary outcomes included day of recurrence of CSA, PMA of last caffeine dose,adverse effects, Extrauterine growth restriction, Necrotizing Enterocolitis, Bronchopulmonary dysplasia, Retinopathy Of Prematurity, and mortality. Results 120 infants were randomized: 60 to each group. There was no difference in the baseline characteristics between the 